\title{
Colombia ¿un líder en la configuración del orden latinoamericano?*
}

\section{Colombia ia leader in the configuration of the Latin American} order?

\author{
Paula Sofía Pedraza Rozo"
}

Fecha de recepción:1/08/2017

Fecha de aceptación: 18/09/2017

\section{Resumen}

Colombia es un país reconocido a nivel internacional por la lucha contra el narcotráfico y terrorismo en el marco del conflicto interno, la innovación y contantes capacitaciones en seguridad y defensa en pro de los intereses nacionales, además de su comercio activo en materias primas y recursos energéticos. Sin embargo, el papel de Colombia en la región se encuentra limitado por la diversidad y heterogeneidad de sus vecinos, reflejado en los distintos proyectos políticos de integración regional (regionalismo), basados en los intereses e ideales de los países miembros y no en un bien común

* Articulo de investigación científica que explora el papel de Colombia en los escenarios de integración latinoamericana

** Profesional en Relaciones Internacionales y semillerista de la Universidad de San Buenaventura, Bogotá. Semillero Individuo, Sociedad y Sistema Internacional. Correo electrónico: sofip_0594@hotmail.com 
en el cual converjan. En este contexto, se busca analizar el rol y posición -liderazgo- de Colombia en la configuración del orden regional; una región que se encuentra fragmentada y dispersa. Así, se toma como marco explicativo los conceptos teóricos de identidad y autoimagen desde el constructivismo, para identificar las fortalezas y debilidades de Colombia en los escenarios regionales de UNASUR y Alianza del Pacífico. Por lo anterior, es pertinente referenciar los cambios y continuidades en materia de política exterior durante el siglo XXI, que han determinado su rol y posición regional, a pesar de que su liderazgo, sigue siendo limitado para la configuración del orden regional latinoamericano. Se reconoce a Colombia como un actor estratégico y clave a partir de sus capacidades y sobre todo, sus potencialidades en el marco de la variabilidad de acontecimientos que acompañan este análisis regional.

\section{Palabras clave}

Política exterior colombiana, América latina, UNASUR, Alianza del Pacífico.

\section{Abstract}

Colombia is a country that is recognized internationally for the fight against drug trafficking and terrorism in the context of internal conflict, innovation and constant training in security and defense for national interests, in addition to its active trade in raw materials and energetic resources. However, Colombia's role in the region is limited by the diversity and heterogeneity of its neighbors, reflected in the various regional integration (regionalism) political projects based on the interests and ideals of the member countries and not on a common good in Which converge. In this context, we seek to analyze the role and position - leadership - of Colombia in the configuration of the regional order; A region that is fragmented 
and dispersed. Thus, the theoretical concepts of identity and selfimage from Constructivism are taken as an explanatory framework to identify the strengths and weaknesses of Colombia in the regional scenarios of UNASUR and Pacific Alliance. Therefore, it is pertinent to refer to the changes and continuities in foreign policy during the XXI century that have determined its regional role and position, despite the fact that its leadership remains limited in the Latin American regional order. Colombia is recognized as a strategic and key player based on its capabilities and above all its potentialities within the framework of the variability of events that accompany this regional analysis.

\section{Key words}

Colombian foreign policy, Latin America, UNASUR, Pacific Alliance

\section{Introducción}

América latina se caracteriza por ser una región periférica, en donde la mayoría de sus países enfrentan crisis y conflictos internos que limitan y han limitado una efectiva proyección e inserción internacional. Sumado a los grandes desafíos nacionales, coyunturales y estructurales, se suman los desafíos que se encuentran en el escenario latinoamericano y que en muchos de los casos no ha permitido determinar y plantear un consenso regional, frente a las grandes amenazas y oportunidades del sistema internacional, que permita hablar de una agenda latinoamericana y gobernanza regional.

Este orden latinoamericano se ha visto definido por la diversidad ideológica, de liderazgos, así como de propuestas de modelos de desarrollo (Torres, 2013). En este contexto, coexisten países que apuestan por propuestas con un enfoque político-social o meramente económico; los primeros, países con gobiernos de izquierda 
y políticas socialistas; por otra parte, se encuentran países que se caracterizan por una fuerte apuesta por el libre mercado. Con base en lo anterior, ha surgido un debate académico que desde distintas perspectivas plantean, por un lado, una América latina diversa y heterogénea en su conformación y estructura (Tokatlian, 2012); por el otro, una región fragmentada frente a la diversidad de proyectos, liderazgos y modelos (Briceño, 2013).

Dentro de este escenario latinoamericano, en términos de integración, el regionalismo latinoamericano se caracteriza por la diversidad en el que varían los objetivos, la institucionalización y la participación estatal; por el solapamiento frente a la condición de miembro que un mismo Estado combina en varias agrupaciones regionales y la doble militancia; por el impulso intergubernamental debido a que los procesos de integración regional han surgido de iniciativas gubernamentales, conducido por las estancias estatales; por el protagonismo empresarial con la participación del sector privado en el impulso y desarrollo de las iniciativas para la ampliación geográfica; y, por ser una estrategia de desarrollo que no impulsa el multilateralismo sino que se limita a complementarlo, priorizando las economías de la región y solo si avanza la liberalización multilateral se mantendrá la compatibilidad con el multilateralismo (Ibañez, 2000). Autores como Achugar (1996), resaltan que cada uno de los regionalismos poseen dinámicas propias y que, por lo mismo sus identidades, tanto locales como supranacionales, viven en constantes transformaciones y/o adaptaciones, dependiendo de su entorno.

Colombia se caracteriza por sus múltiples pertenencias debido a ubicación estratégica en América latina: andino, atlántico, caribe, amazónico, ecuatorial, de la cuenca del Pacífico, occidental, del sur del planeta, de la Orinoquia, americano, latinoamericano, suramericano, iberoamericano y mesoamericano (Carvajal, 2012). Sin embargo, para la primera década del siglo XXI, Colombia enfrentó una 
posición de aislamiento del escenario regional que se acentuó dado que se percibía a Colombia «con una enorme desconfianza no solo en la 'nueva izquierda' sino en el mismo establecimiento de Estados Unidos» (Ardila, 2011, pp. 102).

Con el cambio y transición de política exterior de Juan Manuel Santos, Colombia se caracterizó por ser una potencia regional secundaria, convirtiéndose en uno de los actores fundamentales para la configuración del orden regional. Así, Colombia ha buscado desarrollar una política multilateral participando en distintos escenarios y organizaciones, como la Comunidad Andina de Naciones (CAN), la Unión de Naciones Suramericanas (UNASUR), la Alianza del Pacifico y la Comunidad de Estados Latinoamericanos y del Caribe (CELAC), entre otros.

La UNASUR es un organismo creado a partir del interés geoestratégicos de Brasil y su proyecto político regional, debido a la necesidad de una identidad regional, con énfasis según su objetivo general en la «unión en lo cultural, social, económico y político entre sus pueblos, otorgando prioridad al diálogo político, las políticas sociales, la educación, la energía, la infraestructura, el financiamiento y el medio ambiente» (UNASUR,2008, p.2). Para el año 2008, momento de su creación, América latina se encontraba en el contexto mundial de la crisis financiera, en donde los 12 países que componen la Unión de Naciones Suramericanas (Argentina, Bolivia, Brasil, Chile, Colombia, Ecuador, Guyana, Paraguay, Surinam, Uruguay y Venezuela), se vieron afectados por la reducción del comercio y la disminución de las inversiones extranjeras, por lo que decidieron crear la UNASUR, para afianzar sus vínculos regionales a partir de temas múltiples, que beneficiarán a cada uno de sus miembros (Ocampo, 2009).

Por otra parte, se encuentra la Alianza del Pacífica (AP), la cual se creó a partir de la necesidad de crear vínculos económi- 
cos y comerciales entre países democráticos, que incentivarán el neoliberalismo y el libre mercado (Chile, Colombia, México y Perú). Para los años 2011 y 2012, América latina se encontraba viviendo la recuperación de las crisis financieras y cada uno de los países buscaba minimizar los problemas de desigualdad entre sus sociedades, además, de incentivar el mercado exterior por medio de ayudas estatales (CEPAL, 2011). En este contexto económico y dado el escenario político regional de la primera década del siglo XXI, caracterizada por el Giro a la izquierda latinoamericano, surge la AP

cuyo objetivo es "(...) impulsar un mayor crecimiento, desarrollo y competitividad de las economías de las Partes, con miras a lograr un mayor bienestar, la superación de la desigualdad socioeconómica y la inclusión social de sus habitantes (...)" (Acuerdo Marco de la Alianza del Pacífico, 2012, p.4).

Lo anterior, a partir de la creación de un área de integración profunda, enfocada hacia el Asia Pacífico y que beneficie a cada una de las partes.

En este marco de sub regionalismos, Colombia ha buscado participar en la configuración del orden regional; sin embargo, su participación en cada escenario regional ha estado determinada por factores internos y externos, así como por las debilidades estructurales en materia de política exterior. Así, dada la heterogeneidad regional a partir de la diversidad de intereses nacionales de cada uno de los Estados, los diferentes liderazgos y propuestas de modelos de desarrollo, que han dado paso a nuevas asociaciones y configuraciones de alianzas entre países con afinidades políticas e ideológicas, impactando en la configuración del orden latinoamericano, se plantea como pregunta de investigación ¿̇uál ha sido la participación de Colombia en los escenarios multilaterales de UNASUR y Alianza del Pacífico desde su rol, posición y política exterior para la configuración del orden regional latinoamericano en el los años de 2010 a 2016? 
La metodología parte de un enfoque cualitativo que busca describir las características que posee la región, a partir de las distinciones que surgen entre heterogeneidad y fragmentación que distinguen a la región y han demarcado el contexto que vive cada uno de los países de la zona. Asimismo, se definen los conceptos de rol, posición e identidad que se le atribuyen a los Estados; estos conceptos son apoyados en la teoría constructivista para su aplicación en el estudio de caso colombiano. El método es inductivo, ya que, a partir de la observación de variables como política exterior e integración regional, se busca llegar a una hipótesis y/o conclusión, donde se logre identificar la participación de Colombia en la configuración de la región de América latina.

El diseño metodológico es descriptivo-correlacional, ya que se describe la política exterior colombiana durante el periodo 2010 a 2016, a través de tres categorías de análisis (identidad, rol y posición), para identificar los cambios y continuidades en política exterior, que en parte han determinado su participación en escenarios regionales como UNASUR y Alianza del Pacifico.

Los instrumentos de recolección de información son fuentes primarias y secundarias como lecturas, fichas bibliográficas y de contenido, proyectos con temas específicos sobre Política Exterior y la participación de Colombia en escenarios regionales, además de artículos académicos. Debido a que se necesita tener en cuenta los antecedentes de los temas a investigar, además del apoyo teórico para poder resolver el planteamiento del problema y complementar las descripciones de las categorías.

\section{Política exterior colombiana. Debilidades y fortalezas para su rol y posición regional}

Se ha resaltado la participación limitada de Colombia a través de su política exterior en el ordenamiento regional, debido a que 
todo el siglo XIX e inicios del XX, el país tenía una política exterior orientada hacia el norte (respice polum), debido a que sus intereses nacionales beneficiaban a su socio más cercano, Estados Unidos, el cual por medio de Colombia, buscaba expandir su zona de influencia, no solo en regiones como el Caribe, por medio de la independencia de Panamá, sino en América del Sur con la ayuda de Colombia (Drekonja, 2011).

Se debe tener en cuenta que en el gobierno de Álvaro Uribe (2002 - 2010) las relaciones Colombia - Estados Unidos estaban en auge, gracias al Plan Colombia y la ayuda unilateral brindada por el país del norte, frente al problema del narcotráfico y el conflicto interno colombiano (Ardila, 2012). Además, de que la securitizacion, influenciada por Uribe, enfocada en la lucha contra el terrorismo, impulsada por el contexto interno del país, afianzó las relaciones bilaterales entre ambos países, lo que limitó el papel de Colombia en la región y con otros países semejantes a él (Pastrana y Vera, 2013).

Sin embargo, para la segunda década del XXI, la política exterior colombiana, caracterizada por su orientación hacia el norte, se estaba viendo modificada, no solo por la diversificación de intereses entre ambos países (Colombia - Estados Unidos), sino por el interés de Colombia en establecerse como potencia regional tanto en el Caribe como en la región latinoamericana. Este cambio de política exterior se vio reflejado en la creación del grupo de Contadora con México y Venezuela, a partir de la búsqueda de la paz en Centroamérica, además, de su participación en los Países No Alineados, estableciendo la doctrina (respice similia) (Drekonja, 2011).

Esta nueva política exterior enfocada en el multilateralismo, además de la creación y fortalecimiento de alianzas con otros países, fue generada por la transición en el gobierno y la nueva mentalidad que estaba en el poder colombiano. Cabe resaltar que, con Uribe, Colombia era el país rezagado de Sur América, pero con Juan Ma- 
nuel Santos, Colombia se convirtió en un país colaborador, cooperador e impulsador de una integración regional favorable para todos; en donde, no solo las grandes potencias se verían beneficiadas sino todos los países de la región tuvieran participación (Ardila,2012). Casos como el relanzamiento de la Organización del Tratado de Cooperación Amazónica (OTCA) junto a Brasil en el año 2010, la incursión en Asia a partir de la creación de la Alianza del Pacífico en el año 2012, la búsqueda de la paz en la región a partir de las negociaciones con las Fuerzas Armadas Revolucionarias de Colombia (FARC) y los nuevos tratados bilaterales con la Unión Europea y Corea han caracterizado la nueva política exterior de Colombia, frente a el mundo y la región.

Es así que surge el reconocimiento de Colombia como potencia regional secundaria, en donde autores como Ardila (2012) resaltan el papel de la cancillería y la diplomacia colombiana, en pro de las nuevas relaciones bilaterales y multilaterales con otros países, el mantener la imagen de país democrático, legítimo y trasmisor de confianza, además de impulsor de la economía, bases de un poder blando (soft power), usado por el país latinoamericano para proyectarse como socio fiable, frente a los demás países.

Se podría decir que esta nueva política exterior ha favorecido a Colombia en cuanto economía, comercio, inversión y nuevas alianzas, ampliando su papel como actor regional y como actor internacional, resaltando como fortaleza ese nuevo multilateralismo neoliberal y democrático que ha creado nuevas oportunidades para reconocer a Colombia como actor internacional e impulsador de una integración regional favorable. Sin embargo, una de las debilidades tanto en los países de América latina como en Colombia, es esa diversidad de ideologías que no permiten una unión regional favorable; es así que los países fomentadores del libre mercado están en una parte a favor de la izquierda, de otra parte y los países que piensan 
en ellos mismos; por lo que se resalta que para las siguientes décadas las potencias regionales secundarias, entre ellas Colombia, amplíen su margen de acción y favorezcan a la región vinculándose con las potencias regionales como Brasil y México, que constituyen un pilar clave para la integración.

\section{Colombia en América Latina. ¿Un líder reticente?}

La posición y rol de Colombia en la configuración del orden regional ha sido tema de gran envergadura en los últimos años, de tal forma que, a partir de las dimensiones de concertación, cooperación e integración a nivel internacional, el país suramericano ha venido influyendo en América latina y su posición ha permitido el reconocimiento de Colombia como potencia regional y, en algunos casos, como socio fundamental en aspectos comerciales y económicos.

Autores como Ardila (2012) resaltan la transición del gobierno colombiano como una inserción internacional favorecedora, a pesar de que a finales del gobierno de Álvaro Uribe, el país había empezado a recuperar terreno internacional con el re-apertura de embajadas en el sudeste asiático, además de una fuerte participación en asuntos regionales, como el apoyo en el retorno de Honduras a la Organización de Estados Americanos (OEA), al mismo tiempo de iniciar diálogos con sus países vecinos para reestablecer relaciones. Sin embargo, con Juan Manuel Santos, el papel de Colombia ha ido avanzando, no solo a nivel regional con sus vecinos, sino a nivel internacional como potencia regional secundaria.

En cuanto al papel de Colombia como potencia regional secundaria, Ardila (2012) recalca la constitución de alianzas con líderes regionales como Brasil o México, como uno de los primeros aspectos en cuanto al equilibrio del poder regional. Se debe tener en cuenta que para que un país se categoricé como potencia regional secundaria debe implementar en su mayoría del poder suave (soft 
power) en su política exterior, es decir, que sea un país democrático, donde se demuestre la legitimidad en su gobierno y que así mismo, genere la confianza necesaria a sus países vecinos. En el caso de Colombia, el haber sido catalogado como un estado fallido, con dificultades políticas, sociales y económicas, le permitió restablecer su imagen a través de las negociaciones de paz; así mismo, crear nuevas alianzas con otros actores, siendo catapultado como un país atractivo para la Inversión Extranjera Directa (IED). Es así que, gracias a el reposicionamiento del país suramericano y sus nuevos ideales multilateralitas, ha ampliado su margen de acción en Centroamérica y en Suramérica, catalogándose como potencia regional secundaria.

Adicional a su nueva visión planteada por Juan Manuel Santos, se debe tener en cuenta las herramientas de política exterior aplicadas por Colombia para el mantenimiento de este nuevo orden regional, casos como la diplomacia económica y la cooperación Sur - Sur en cuanto a temas de seguridad han permitido el avance Colombia en los últimos años. En cuanto a la diplomacia económica, se ha caracterizado por ser una diplomacia personalizada y presidencialista, en donde el presidente es el principal actor a la hora de realizar visitas a otros estados, teniendo en cuenta el pilar de su gobierno, que es posicionar a Colombia como actor influyente a nivel regional, como a nivel internacional, además de la necesidad primordial de que Colombia reciba inversiones económicas y de innovación, para potencializar el comercio y la industria nacional.

En cuanto a la cooperación Sur - Sur se ha dado en aspectos de seguridad y medio ambiente, al ser Colombia uno de los países con mejor formación en fuerzas militares, para el mantenimiento de la protección fronteriza y la garantía de una población segura; es por esto que la cooperación con países vecinos en cuanto aspectos de formación en seguridad ha cobrado fuerza la última década, proyectando a Colombia como un escenario de enseñanza y de formación 
en seguridad. Es así que países como Brasil, Perú y México envían a sus militares a capacitarse en la Escuela Superior de Guerra de Colombia, para que amplíen sus conocimientos y los apliquen devuelta a sus territorios (Cardona, 2011).

Se debe tener en cuenta los conceptos de identidad, rol y posición empleados por el constructivismo para analizar el papel de Colombia en América latina; entendiéndose la identidad como la continuidad entre el liderazgo político y las esferas de influencia según Kiselev y Smirnova (2004). Esta continuidad entre liderazgo e influencia permite estructurar la identidad propia del país, sus principales características en el marco de política exterior como de política interna que distingan al estado de otros estados. Para Colombia, la influencia de cada uno de los presidentes y líderes políticos ha generado diferentes concepciones de lo que es la identidad del país; se resalta que la política exterior colombiana es de gobierno y no de estado.

Según Pastrana y Vera (2013), la política exterior colombiana ha venido transformándose desde el año 2010 con el cambio de gobierno, en donde la transición del gobierno se mantuvo enfocada en los tres pilares de política exterior creados en el gobierno de Uribe y sucedidos por el gobierno de Santos, 1. la internacionalización de la economía, 2. la diversificación en las exportaciones y, 3. el incremento de la inversión, siendo los únicos rasgos estructurales continuos en ambos gobiernos. Sin embargo, y, para el cambio de gobierno, la proyección y lineamientos de política exterior están impulsados por la diversidad geográfica y ya no tanto por los tres pilares dejados por su antecesor. En donde, el Presidente Santos busca que Colombia tenga un rol internacional; esta nueva identidad va permeada por los lineamientos de política exterior para ambos periodos (2010 - 2014, 2014 - 2018), los cuales le permiten tener un actuar más amplio en lo que se refiere a Colombia en la agenda internacional: 1. energía, 
2. educación, 3. telecomunicaciones y tecnologías de la información, 4. innovación, 5 . ciencia y tecnología, 6 . biodiversidad y cambio climático, 7. desarrollo agrícola, 8. infraestructura y, 9. comercio e inversión (Pastrana y Vera, 2013).

En consecuencia, la identidad de Colombia en cuanto a su política exterior refleja una política de gobierno y no de estado, ya que no ha existido una consecución en cuanto a la proyección del país en la región. Es así que cada gobierno, al llegar al poder determinar el accionar de sus políticas en favor de sus propios intereses y la variabilidad de sus esferas de influencia y de poder. Del mismo modo, la identidad de un estado afecta la construcción de sus intereses, tanto nacionales como en política exterior, lo que permite que el país cree interacciones con otros estados en la búsqueda de una identidad estatal, haciendo referencia a la variabilidad en identidades que poseen las naciones gracias a la variabilidad en sus mandatarios (Hopf, 2002).

Se puede decir que en cuanto a la creación de una identidad en una política exterior de gobierno se debe tener en cuenta aspectos en común que tienen los mandatarios a la hora de posicionarse, además aspectos históricos que permiten mantener ciertos lineamientos en la política exterior. Como es el caso del conflicto interno en Colombia, en donde todos los mandatarios mantenían sus políticas de seguridad y cooperación, ya fuese de manera bilateral o multilateral para la lucha contra las amenazas internas y que pudieran afectar a los países vecinos.

En cuanto al rol, Kiselev y Smirnova (2004), lo definen como el papel o imagen situacional que tienen los estados, que buscan crear o mantener un liderazgo político en un espacio y tiempo específicos, relacionándose con la concepción de que los estados son actores sociales. Es decir, cada país tiene un objetivo y un fin propio, frente a su política exterior $\mathrm{y}$, por lo tanto, las acciones generadas por 
los estados son los medios para crear su rol o papel internacional. Para el caso colombiano, este rol se ha visto modificado, al mismo tiempo que se ha cambiado de gobierno y, por lo mismo de política exterior.

Colombia a inicios del siglo XXI se ha visto reconocido, no solo por su conflicto interno, o las distintas masacres, violaciones de Derechos Humanos (DD. HH), crímenes de lesa humanidad, maltrato infantil entre otros, sino por ser un país en vías de desarrollo, donde su principal producto es el café y el banano. Sin embargo, y después del 11 de septiembre, el papel de Colombia a nivel regional y a nivel internacional ha ido cambiando; aspectos como la transición en el gobierno de Uribe a Santos y la proyección de Colombia como actor del mundo han llegado a demostrar cuan influyente podría ser el país latinoamericano en la sociedad internacional.

Para Dallanegra (2012), la política exterior de Santos ha sido impulsada por las diferencias ideológicas de cada uno de los países, ya que, a partir de estas distinciones entre las naciones, puede existir una cooperación recíproca y un respeto mutuo. Además de ampliar el campo de visión de Colombia, es decir, no limitarse a un margen de acción Colombia - Estados Unidos sino Colombia - países del mundo. Esta visión es recalcada por el autor en cuanto al aumento en los acuerdos de cooperación e integración firmados por Colombia, 16 en total, que tienen temáticas de infraestructura, mejora en carreteras y oleoductos, comercio, creación de empresas, turismo, lucha antinarcóticos y desarrollo fronterizo, garantizándole a Colombia un rol activo en la región.

En cuanto a la posición de un país, -valoración que los demás países hacen frente a los otros, teniendo en cuenta la concepción propia-, se complementa con el papel o imagen situacional que Kiseley y Smirnova (2004), denominan el rol de un Estado frente a las conductas internacionales explicado anteriormente. En el caso 
colombiano, el país cuenta con una multiplicidad geográfica, empezando por los Andes, Centro América y el Caribe, la Amazonía, el Atlántico y el Pacifico, Suramérica y Latinoamérica entre otros (Carvajal, 2012).

Debido a esta multiplicidad geográfica, Colombia se ha convertido en un corredor transfronterizo, el cual tiene la principal característica de conectar el norte con el sur, creando una fuerte posición en los escenarios multilaterales. Países a través de la historia han visto a Colombia como actor fundamental para la entrada en América del Sur, casos como Estados Unidos y su ayuda en el Plan Colombia, en donde autores como Ortiz (2017) recalcan que, el acercamiento con Estados Unidos y la posición geográfica de Colombia, han limitado su inserción a nivel regional, ya que ha generado una posición geopolítica dual, en donde se ha restringido crear una visión en conjunto de lo que es América latina.

Se podría decir que, a nivel regional, Colombia está dando sus primeros pasos para recuperar y crear una posición y un papel como actor fundamental. Esto se debe a la fuerte transición en el cambio de gobierno, de pasar de ser un país aislado, enfocado en el norte, a un país con política exterior multilateral, impulsador de la cooperación y las nuevas alianzas multitematicas que brindan mayores beneficios para cada una de las naciones.

\section{Colombia en escenarios regionales. UNASUR y Alianza del Pacífico}

Colombia, a partir del gobierno de Juan Manuel Santos ha ido mejorando las relaciones con sus vecinos; sin embargo, el enfoque multilateral con fines hacia Europa y el Pacífico, ha limitado su ac- 
cionar en la región. En un «vecindario fragmentado», como lo es la región latinoamericana existen múltiples escenarios de integración, que han permitido proyectar a Colombia como potencia regional secundaria; sin embargo, esta distinción se vino a dar con Juan Manuel Santos en el poder, ya que al llegar a la Casa de Nariño buscó nuevas alternativas de integración, a través de asociaciones con países semejantes, en el marco de la cooperación sur-sur.

Es así que, en el sistema internacional, los estados, en especial las potencias regionales, pueden definir y perseguir intereses distintos; sin embargo, para mantener el orden en las regiones surgen las potencias secundarias que limitan el poder de la potencia, permitiendo crear un equilibrio y según Pastrana y Vera (2013), creando un freno a las políticas exteriores de las potencias en cada región. Es así como Colombia ha ido creando su rol de actor activo, a nivel regional por medio de su crecimiento económico, sus impulsos en la mejora de infraestructura y en el campo del desarrollo humano y sostenible, que han permitido crear un rol de potencia regional secundaria, brindándole mayor seguridad a la región y a los nuevos socios del país.

Autores como Cardona (2011) explican que el neoliberalismo ha sido el enfoque usado a nivel mundial, en donde la cooperación es el pilar de las políticas exteriores actuales; se priman temáticas como el comercio, el libre mercado y la lucha por los intereses en común sobre los intereses propios. Cabe resaltar que, para inicios del siglo XXI, Colombia ha incrementado su participación a nivel regional, gracias a sus iniciativas en favor de la economía latinoamericana, debido a las crisis financieras vividas entre 2008 y 2012, que afectarían la revaluación de las monedas en la región. Es por esto que el ideal multilateralista expresado por el gobierno Santos, en pro de impulsar a Colombia en tener un rol a nivel regional por medio de alianzas con países con intereses y políticas exteriores semejantes. Casos como la creación de la Alianza del Pacífico con Perú, México y 
Chile o los tratados con la Unión Europea y Corea del Sur, que han permitido el acercamiento de Colombia, a mercados lejanos impulsando grandes vínculos a nivel regional e internacional.

Es así que, escenarios regionales como la Unión de Naciones del Sur (Unasur) o la misma Comunidad de Naciones (CAN) se encuentran obsoletas y se ven como mecanismos de integración fallida (Dallanegra, 2012); esto se debe a la primacía de los intereses nacionales, además de las asimetrías en el poder han debilitado a las organizaciones y no han permitido una integración regional favorable, a través de estas organizaciones. Según Ardila (2012), el papel de Colombia en organismos de integración como Unasur, ha tenido dos momentos; el primero fue en el gobierno del presidente Uribe, en el cual la organización limitó gran parte de las iniciativas bilaterales, propuestas por el mandatario en pro de la inserción de Estados Unidos a la región, lo que redujo la posición de Colombia a nivel regional. Sin embargo, y como segundo momento, la llegada del presidente Santos normalizó las relaciones con la organización y transformó la posición de Colombia en un actor influyente en el escenario, impulsado por diversificar las relaciones con otros regionalismos, crear y fortalecer las alianzas regionales, además de impulsar las mejoras en las relaciones bilaterales con Venezuela.

Se recalca que la concepción propia de Colombia ha sido punto fundamental en la valoración que tienen los países de la región, ya que, la búsqueda de la paz y el fortalecimiento de la seguridad, han promocionado a Colombia como un país líder en el ámbito de seguridad. Es decir, que un aspecto fundamental como la búsqueda de la paz por parte de gobierno a través de los lineamientos de política exterior han permitido crear una posición en temas de seguridad por parte de Colombia a nivel regional. Además del desarrollo en el campo de integración energética por medio de los biocombustibles y la propuesta de crear un gaseoducto que conecte Venezuela, Co- 
lombia y Panamá permitiendo una futura reducción de costos en el transporte del gas y la gasolina (Ardila, 2012).

En relación a la Alianza del Pacífico, se debe tener en cuenta que es un mecanismo de integración joven, en donde se resalta su baja integración económica, dado que entre sus miembros el intercambio es mínimo, debido a que producen productos similares; sin embargo, en cuanto a integración social, según Ortiz (2017), la AP es constituida como una entidad cultural y geoestratégica enfocada en el neoliberalismo, aplicada a la creación de un área de libre comercio, cuyo fin es una integración profunda dentro de una región latinoamericana, en pleno desarrollo y construcción. Se debe tener en cuenta que la AP como mecanismo de integración ha ido progresando, apoyándose en el principio del libre tránsito de personas, servicios, capitales y bienes, que es el mayor paso para lograr bienestar social e inclusión para todos los ciudadanos de los cuatro países (Ortiz, 2017).

Se podría decir que, la búsqueda en la mejora del posicionamiento de Colombia a nivel regional, se ha caracterizado por ser transversal en cuanto a sus políticas internas y sus políticas exteriores, ya que han ejercido un fuerte impulso en la valoración de los vecinos frente a Colombia. Casos como el reconocimiento como potencia regional secundaria, el posicionamiento de Colombia como país CIVETS, agrupándolo con economías emergentes de diferentes regiones, que proyectan un desarrollo con miras a convertirse en una economía fuerte y estable, además de manejar un poder blando (soft power), influenciado por la democracia, la legitimidad y la confianza, permitiendo que Colombia y su participación en el orden regional sea equilibrada, tratando de no alterar la estructura regional.

\section{Reflexiones Finales}

El papel de Colombia en América latina ha ido mejorando en la última década, debido a los pequeños acercamientos favorables con 
sus vecinos, no solo en temas comerciales y económicos, sino en el marco ambiental, social, de seguridad e infraestructuras entre otros. Sin embargo, la falta de proyección en la región ha venido limitando la valoración de los países vecinos, lo que ha generado una baja estructura en el orden regional, pues no se ha enfocado en políticas comunitarias y multilaterales que favorezcan a la región, por medio de actores como Colombia y Chile que han venido ganando un reconocimiento a nivel internacional, tanto por su estabilidad como por la búsqueda de la paz. Sino que se ha enfocado en políticas unitarias que favorecen a los intereses propios de los países y no permiten la creación de una región formal.

Es por esto y a pesar del fuerte crecimiento y proyección de Colombia como actor regional, que su proyecto de regionalismo no ha ido avanzando mucho. Ya sea a partir de la Alianza del Pacifico, como actor regional proyectado a los países asiáticos o la UNASUR como mecanismo de integración regional, a partir de la seguridad transfronteriza, la región de América Latina no se constituirá fácilmente debido a la diversidad y fragmentación en la que se encuentra. Sin embargo, y, con las nuevas transiciones en los gobiernos suramericanos, la democracia y el neoliberalismo podrían ser puntos clave para la creación de un orden regional latinoamericano, que impulse tanto a Colombia como a la región, como principales actores en el sistema internacional.

Eso sí, para Colombia el siglo XXI ha ido favoreciendo al país ya sea en temas económicos, comerciales, sociales y regionales, ya que su rol a nivel regional, cada vez incrementa más y con la política exterior de Juan Manuel Santos, enfocada en la proyección de Colombia como actor regional e internacional, el país crecerá de manera favorable, tanto para impulsar a la región como mejorar internamente la calidad de vida de sus ciudadanos. Se debe resaltar la agenda multilateral del país en pro de mejorar las relaciones con sus 
países vecinos como con países lejanos a la esfera regional. Es por esto que mecanismos como la Alianza del Pacífico son una plataforma para la proyección e inserción de la región de América latina en el mundo, además, que establece relaciones con otras regiones del mundo, permitiendo un reconocimiento de los países suramericanos en otros espacios e incrementan la participación de la región en nuevos espacios de integración; es así que Colombia y su papel regional son factores claves para América latina y su crecimiento.

\section{Referencias Bibliográficas}

Achugar, H. (1996). Repensando la heterogeneidad de Latinoamericana. Revista Iberoamericana, 62 (166-167), pp. 845-861. Uruguay: Universidad de la Republica.

Alianza del Pacífico. (2012). Acuerdo Marco de la Alianza del Pacífico. Recuperado de: https://alianzapacifico.net/?wpdmdl=4464

Ardila, M. (2012). Potencia regional secundaria en definición: Colombia entre Sur y Centroamérica. Papel Político, 17(1), 293-319.

Ardila, M. (2011). Prioridades e instrumentos de la inserción internacional de Colombia. En Cardona, D. Colombia: una política exterior en transición. Bogotá: Friedrich Ebert Stiftung en Colombia (Fescol)

Briceño, J. (2013). Ejes y modelos en la etapa actual de la integración económica regional en América Latina. Revista de Estudios Internacionales,175, pp. 9-39. Instituto de Estudios Internacionales, Universidad de Chile.

Cardona, D. (2011). Seguridad y política exterior en la gran región andina: reflexiones y escenarios. En Colombia: Una política exterior en transición, 1, pp. 3 - 32. Fundación Friedrich Ebert en Colombia (Fescol): Bogotá

Carvajal, L. (2012). Colombia: País Puente en Política Exterior: retos y desafíos. Centro de pensamiento estratégico, pp.1-17. Bogotá: Ministerio de Relaciones Exteriores

CEPAL. (1970). La economía de América Latina en 1969. Publicación de Naciones Unidas, pp. 1-420. Nueva York: Estados Unidos.

CEPAL. (2011). Panorama social de América Latina. Publicación de Naciones Unidas, pp. 1-253. Santiago de Chile: Chile. 
Constitución Política de Colombia. (1991). Constitución Política de Colombia, pp.18. Bogotá: Colombia

Dallanegra, L. (2012). Claves de la política exterior de Colombia. Revista Latinoamerica, 1, pp. 37-73.

Drekonja, D. (2011). Formulando la política exterior de Colombia. En Borda, S. y Ticker, A. (Compiladoras), Relaciones internacionales y política exterior de Colombia (pp. 4978). Bogotá, Colombia: Ediciones Uniandes

Hopf, T. (2002). Social construction of international politics: identities \& foreign policies. Cornell University Press.

Ibáñez, J. (2000). El nuevo regionalismo latinoamericano en los años noventa. Electrónica de Estudios Internacionales, 1, pp. 1-11. Chile: CEPAL

Kiselev, I., Smirnova, A. (2004). La dinámica de la autoimagen de los Estados Unidos a través de los discursos públicos. Psicología Política, 28, pp. 55-68. Universidad Estatal de Yaroslavl, Rusia.

Ocampo, J. (2009). Impactos de la crisis financiera mundial sobre América Latina. Revista CEPAL, 97, pp. 9-32. CEPAL

Ortiz, C. (2017). La Alianza del Pacífico como actor regional: factores de éxito para la cohesión regional hacia la proyección internacional. Desafíos, 29(1), pp.49-77. Doi: http://10.12804/revistas.urosario.edu.co/desafios/a.4885

Pastrana, E. y Vera, D. (2013). Las relaciones entre Colombia y Brasil en un contexto de regionalización diversificada en Suramérica y de un mundo multipolar emergente. Papel Político, 18(2), pp. 613-650. Pontificia Universidad Javeriana: Bogotá

Tokatlian, J. (2012). La política exterior de América Latina: ¿Un reordenamiento? Centro de Pensamiento Estratégico, pp. 3-19. Recuperado de: https://www.yumpu.com/es/ document/view/14238201/politica-exterior-un-reordenamiento-de-america-latina

Tokatlian, J. (2013). América latina y su gran estrategia: entre la aquiescencia y la autonomía. CIDOB d'Afers Internacionals, 104, pp. 157-180.

Torres, M. (2013). Las Políticas regionales de México, Brasil y Venezuela con respecto a la integración de América Latina. Invenio: Revista de investigación académica 30, pp. 29-46. Recuperado de: https://dialnet.unirioja.es/servlet/articulo? $\operatorname{codigo}=4233848$

UNASUR. (2008). Tratado Constitutivo de la Unión de Naciones Suramericanas. Recuperado de: http://www.cancilleria.gov.co/sites/default/files/tratado-constitutivo-unasur.pdf 